\title{
SOME RESULTS ON ONE-RELATOR GROUPS
}

\author{
BY B. B. NEWMAN
}

Communicated by L. Auslander, January 11, 1968

The results in this note arose from considering the question: What are the abelian subgroups of a one-relator group? The additive group of $p$-adic rationals and the free abelian group of rank 2 are certainly subgroups of a one-relator group. For example in

$$
G=\operatorname{gp}\left\{a, b ; a^{-1} b^{2 p} a b^{-2}\right\}
$$

the infinitely generated subgroup

$$
H=\operatorname{sgp}\left\{b^{2}, a^{-1} b^{2} a, a^{-2} b^{2} a^{2}, \cdots\right\}
$$

is isomorphic to the additive group of $p$-adic rationals, and

$$
K=\operatorname{sgp}\left\{b^{2}, b^{-1} a^{-1} b a\right\}
$$

is free abelian of rank 2. In 1964 Gilbert Baumslag [1] conjectured that the additive group of rationals is not a subgroup of a one-relator group. That this conjecture is correct follows from the following theorem.

THEOREM 1. Let $G$ be a torsion-free one-relator group. Then no nontrivial element of $G$ has more than finitely many prime divisors. Moreover a nontrivial element is not divisible by more than finitely many powers of a prime $p$ if $p$ is greater than the length of the relator.

REMARK. An element $g$ of a group is divisible by an integer $n$, or has a divisor $n$, if $g$ has an $n$th root in the group. By the length of the relator is meant the letter length of the relator as a word in a free group.

R. C. Lyndon [7] has shown that the cohomological dimension of a torsion-free one-relator group is $\leqq 2$. Now the cohomological dimension of a free abelian group of rank $n$ is $n$, and of a direct product of an infinite cyclic group with a noncyclic locally cyclic group is $>2$, (see [6], [2]). Since the cohomological dimension of a subgroup is less than or equal to the cohomological dimension of the group, it follows that the only abelian subgroups of a torsion-free one-relator group are free abelian of rank $\leqq 2$ or locally cyclic subgroups in which every nontrivial element is divisible by at most finitely many primes.

The proof of Theorem 1 uses the usual argument of the Freiheitssatz (see [9]) together with the following ideas.

Definition. Let $H$ be a subgroup of $G$ and $p$ a prime. Then $H$ is 
$p$-pure in $G$ if for each $g \in G$ and positive integer $r, g^{p^{r}} \in H$ implies that there exists an element $h \in H$ with $g^{p^{r}}=h^{p^{r}}$.

Lemma 1. Let $C=\{A * B ; J\}$ be the generalized free product of two groups $A, B$ amalgamating a subgroup $J$. If $J$ is a p-pure subgroup of the factors $A, B$ then $A, B$ are p-pure subgroups of $C$. Moreover a nontrivial element of $C$ is divisible by all powers of the prime $p$ only if a nontrivial element of $A$ or $B$ is divisible by all powers of the prime $p$.

The concept of a $p$-pure subgroup is the appropriate tool for proving Theorem 1, and indeed the whole argument turns on the following key lemma.

Lemma 2. Let $G$ be a one-relator group. Then any subset of the generators of $G$ generates a p-pure subgroup of $G$ where $p$ is any prime greater than the length of the relator.

For one-relator groups with elements of finite order one can say much more.

THEOREM 2. Let $G$ be a one-relator group with torsion. Then the centralizer of every nontrivial element of $G$ is cyclic.

The proof of Theorem 2 is similar to the proof of Theorem 1 except instead of using $p$-pure subgroups one uses $\mu$-subgroups defined as follows:

Definition. Let $H$ be a subgroup of $G$. Then $H$ is a $\mu$-subgroup of $G$ if for all $g \in G$,

$$
g^{-1} H g \cap H \neq 1 \text { implies } g \in H .
$$

Lemma 3. Let $C=\{A * B ; J\}$ where $J$ is a $\mu$-subgroup of the factors $A, B$. Then $A, B$ are $\mu$-subgroups of $C$. If in $A, B$ the centralizer of every nontrivial element is cyclic, then in $C$ the centralizer of every nontrivial element is cyclic.

LEMMa 4. Let $G$ be a one-relator group with torsion. Then any subset of the generators of $G$ generates a $\mu$-subgroup of $G$.

The proof of Lemma 4 is not easy and depends on the following seemingly powerful result.

TheOREM 3. Let $G=\operatorname{gp}\left\{a, b, \cdots ; R^{n}\right\}, n>1$, where $R$ is cyclically reduced. Suppose that two words $W=W(a, b, \cdots), V=V(b, \cdots)$, where $W$ is a freely reduced word containing a nontrivially and $V$ does not contain a, define the same element of $G$. Then $W$ contains a subword which is identical with a subword of $R^{ \pm n}$ of length greater than $(n-1) / n$ times the length of $R^{n}$. 
This result tells us something about the actual spelling of words representing elements of $G$.

COROLLARY 1. The word problem and the extended word problem are solvable in $G$.

The proof of this result by W. Magnus (see [9]) is a rather complicated process and it does not show that in the special case of less than $\frac{1}{6}$ groups (see [3]) a much simpler solution is possible. Theorem 3 , however, provides the simplest of algorithms.

CoRollary 2. Let $F$ be a free group on a set $X$ of generators and let $r \in F$ and $N=\left\{r^{n}\right\} F, n>1$. Let $G$ and $H$ be generated by subsets $Y$ and $Z$ of $X$. Then $G H N$ is a recursive subset of $F$.

This solves a problem of R. C. Lyndon (see [8, Problem 3.6]) in the case where $N$ is the normal closure in $F$ of a proper power. Lyndon points out that a solution of this problem enables one to considerably extend the Magnus solution of the word problem for one-relator groups.

The Freiheitssatz for groups with torsion is an immediate consequence of Theorem 3, and may be generalized in several directions, for example:

Corollary 3. Let $H=\operatorname{sgp}\left\{a^{\beta}, b, \cdots\right\}$ be a subgroup of $G$ where $a$, $b$ are nontrivial in $R$. Then $H$ is a free group freely generated by $a^{\beta}$, $b, \ldots$ if $\beta$ is any integer $>2 \alpha$ where $\alpha$ is the largest absolute value of an a-exponent in $R^{n}$.

This extends a result of N. S. Mendelsohn and Rimhak Ree [10].

If $n \geqq 8$ then the group is a less than $\frac{1}{8}$ group and from results of M. Greendlinger [4] the conjugacy problem is solvable in $G$ if $n \geqq 8$. In this direction see also M. Greendlinger [5] for less than $\frac{1}{6}$ groups, and V. V. Soldatova [11] for a subclass of less than $\frac{1}{4}$ groups. By using a generalization of $\mu$-subgroups one may prove

THEOREM 4. The conjugacy problem is solvable in one-relator groups with torsion.

Complete details, extensions, and applications of the results in this note will be submitted in a paper later.

I wish to acknowledge the encouragement given by Gilbert Baumslag.

\section{BIBLIOGRAPHY}

1. G. Baumslag, Groups with one defining relator, J. Austral. Math. Soc. 4 (1964), 385-392. 
2. G. Baumslag and K. W. Gruenberg, Some reflections on cohomological dimension and freeness, J. Algebra 6 (1967), 394-409.

3. M. Greendlinger, Dehn's algorithm for the word problem, Comm. Pure Appl. Math. 13 (1960), 67-83.

4. - On Dehn's algorithm for the conjugacy and word problems with applications, Comm. Pure Appl. Math. 13 (1960), 641-677.

5. - Solution by means of Dehn's generalized algorithm of the conjugacy problem for a class of groups which coincide with their anti-centers, Dokl. Akad. Nauk SSSR 158 (1964), 1254-1256=Soviet Math. Dokl. 5 (1964), 1384-1386.

6. R. C. Lyndon, Cohomology theory of group extensions, Duke Math. J. 15 (1948), 271-292.

7. - Cohomology theory of groups with a single defining relation, Ann. of Math. 52 (1950), 650-665.

8. - Dependence and independence in free groups, J. Reine Angew. Math. 210 (1962), 148-174.

9. W. Magnus, A. Karrass and D. Solitar, Combinatorial group theory, Interscience, New York, 1966.

10. N. S. Mendelsohn and Rimhak Ree, Free subgroups of groups with a single defining relation, Notices Amer. Math. Soc. 14 (1967), 90.

11. V. V. Soldatova, On a class of finitely presented groups, Dokl. Akad. Nauk SSSR 172 (1967), 1276-1277 = Soviet Math. Dokl. 8 (1967), 279-280.

Fairleigh Dickinson University and

University College of Townsville, Queensland, Australia 